\title{
Club Jules Gonin Council Members
}

New Council from January 1st, 1985

\section{President}

Robert Machemer, Durham

Vice-President

Alan C. Bird, London

Secretary

Nicolas Ducrey, Lausanne

Treasurer

Michel Gonvers, Lausanne
Members

Pierre Amalric, Albi

George Blankenship, Miami

Basil Daicker, Basel

Jens Edmund, Copenhagen

T. Juan Verdaguer, Santiago

Achim Wessing, Essen

\section{Honorary Members}

Marc Amsler, Sierre $\dagger$

Hermenegildo Arruga, Barcelona $†$

Norman Ashton, London

Paul Bailliart, Paris $\dagger$

Gianbattista Bietti, Roma $†$

Paul Bregeat, Paris *

José Casanovas, Barcelona

Paul Cibis, St. Louis †

David Cogan, Chevy Chase

Ernst Custodis, Düsseldorf

Marc-Adrien Dollfus, Lyons-la-Forêt $\dagger$

André Dubois-Poulsen, Paris

René Dufour, Lausanne $\dagger$

Stewart Duke-Elder, London $\dagger$

Lorimer Fison, London

Jules Francois, Gent $\uparrow 1984$

Hans Goldmann, Bern

Gabrielle Gonin, Lausanne

Louis Guillaumat, Paris

* elected in 1984

$\dagger$ deceased members

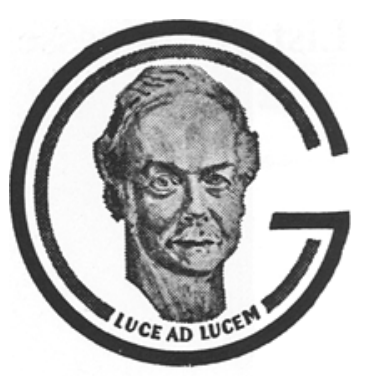

CLUB JULES GONIN
Karl Hruby, Vienna

James R. Hudson, London

Rüdolf Klöti, Zürich *

E. Edward Maumenee, Baltimore

Gerd Meyer-Schwickerath, Essen

Hans-Karl Müller, Bonn $\dagger$

Karl Mylius, Hamburg

E.W.D. Norton, Miami

Louis Paufique, Lyon $\dagger$

D.K. Pischel, San Francisco

Algernon Reese, New York $\dagger$

Bengt Rosengren, Uppsala $\dagger$

Charles L. Schepens, Boston

C. Dee Shapland, Harrow-on-Hill

H.B. Stallard, London $\dagger$

E. Bernardo Streiff, Lausanne

Alberto Urrets-Zavalia, Cordoba *

H.J.W. Weve, Zeist $\dagger$ 